\title{
Recommendations for Setting Backup Stages Line Distance Relay Protection
}

\author{
Tran Hoang Quang Minh \\ Faculty of Electrical and Electronic Engineering, Ton Duc Thang University, Ho \\ Chi Minh City, Vietnam \\ tranhoangquangminh@tdt.edu.vn
}

\section{Abstract}

In this paper, main is analyzing the technical actions to limit and expand the characteristics numerical distance relay protection in order to reduce losses and improve functioning protection, and considering the specifications, efficiency and losses of the backup stages distance relay protection on high-voltage lines. Addition to this, criterion technical efficiency of back-up stage distance relay protection is presented. Based on these analysis and criterion, recommendations for designing and setting backup stages distance relay protection are proposed.

Keywords: Technical efficiency; technical effect; distance relay protection; electric network; backup stage

\section{Introduction}

In the modern world of relay protection (RP), due to electrical networks found metrological advances (inhibition of the currents, the configuration of the characteristics of operating in the complex plane of the resistance, the drop voltage negative and zero sequence on the lines), and circuit solutions almost entirely (differential relay protection) or partially (distance relay protection). These eliminated the refusals of work, false and excessive actions. For relay protection with the exchange information on operation between these ends of lines, the operation principles are spread on the principle of differential relay protection, and achieved similar performance. However, there is not small quantity class steps current and distance relay protection (first line), in which the property is a failure for external faults is achieved by temporary blocking system levels. These actions take place unrecoverable loss of functioning relay protection like: refusals of work, false and excessive actions. These actions are depending on the classification regime -switching states of the network, the types of faults, interference, generally expressed as a function of the selected setting [1-2].

The most importing thing is analyzed and proposed recommendations for designing and setting relay protections. Technical effect of channels (stages) relay protection lines is calculated as the difference between potentially possible effect (the probability of faults on the protected line) and losses (the sum of the probabilities of refusals of work, false and excessive actions). And technical efficiency is quotient of technical effect to the potentially possible effect is a very full measure of the quality operation of relay protection. Introduction, description, formulas for determining the technical efficiency, technical effects and losses of distance relay protections high-voltage lines were presented in [1-3,5], and were developed in [8-9].

In this paper, main ideas are discussed the analysis of technical measures to control and enhance the characteristics of digital relay resistance distance relay protection in order to reduce losses and improve the functioning of these protections. Detailed features, performance and operation of the losses backup stages of distance relay protections of high-voltage lines are considered and studied. Addition to this, criterion technical efficiency of back-up stage distance relay protection is presented and analyzed. 
Based on these, the recommendations for functioning and setting backup stages of highvoltage lines distance relay protections are proposed.

\section{Main Part}

At the present time, in connection with the development of technology, resistance relay of distance protection relays are performed on a digital basis. Digital resistance relay are widely used in electrical networks, so the questions of adaptation and the efficiency of the digital resistance relay and its characteristics in the complex surface, especially the expansion and reduction characteristics to increase the efficiency of distance relay protections. A general view of a digital distance relay protection of high-voltage lines is shown in Figure 1. On the basis of characteristic, the functioning of resistance relay in the digital distance relay protections is analyzed.

Firstly features of characteristic digital relays resistance are ascertained.

1. The basis characteristic in the complex surface $\mathrm{Z}$ digital resistance relay is a parallelogram with the top and bottom faces parallel to the $\mathrm{X}$-axis (the axis of resistances) and the lateral edges at an angle to the horizontal axis is equal or close to the angle of the line resistance. One (upper or lower) bound of a parallelogram is removed from the $\mathrm{X}$-axis by the amount of setting reactance for the first and second quadrants, or third and fourth quadrants of the surface $\mathrm{Z}$, depending on the direction of the resistance relay: towards the protected line (network adjacent to the side of the transformers) or in the opposite direction. The second face is located in the direction opposite quadrants, and usually is located in the quadrants on the other side of the $\mathrm{X}$-axis.

2. To ensure the orientation in the space of opposite quadrants the parallelogram characteristic is limited by lines passing through the origin of coordinates at an angle $\varphi_{2}$ and $\varphi_{3}$ (relatively horizontal axis) and cut the space of the parallelogram, respectively: in the first or fourth quadrants $\left(\varphi_{2}\right)$, the second quadrant $\left(\varphi_{3}\right)$ in the direction of protection to the lines; the second and third quadrants $\left(\varphi_{2}\right)$, the fourth quadrant $\left(\varphi_{3}\right)$ in the direction of protection to the buses. Limiting straight lines under these angles are realized with phase-limiting relay in the surface Z. Two straight phase-limiting lines may well be implemented on a single phase-limiting relay, setting it through the phase meter with a scope less than $180^{\circ}$.

3. Guaranteed measurement of positive-sequence resistance of the relay performs with sinusoidal currents and voltages. However, the sinusoidal currents and voltages may contain occasional free components of the electromagnetic transient process, which can distort the measured resistance. To reduce and eliminate these distortions in the input circuits of the relay resistance set filters that produce forced sinusoidal power-frequency components.

4. Formation characteristic need to account possible regime-switching network status, metrology equipment in the form of the current distribution coefficients, the coefficients of the detuning (approval), the sensitivity with respect to faces of the parallelogram and the phase-limiting straight lines, resulting in needless result to expand or reduce the boundaries of the parallelogram and the changes of angles phase-limiting lines, notably to change the scope of the characteristic.

5. The necessity to change the boundaries of characteristic also occurs during the pass of operating-current through protection in the first moments of the electromagnetic transient process of the faults, as due to inertia of the rotor generators currents continues to transmit over the network. Operating currents are summed up with the forced sinusoidal currents of faults in the same relay protection, and is subtracted in the other. This leads to a decrease or an increase in resistance measurements. 


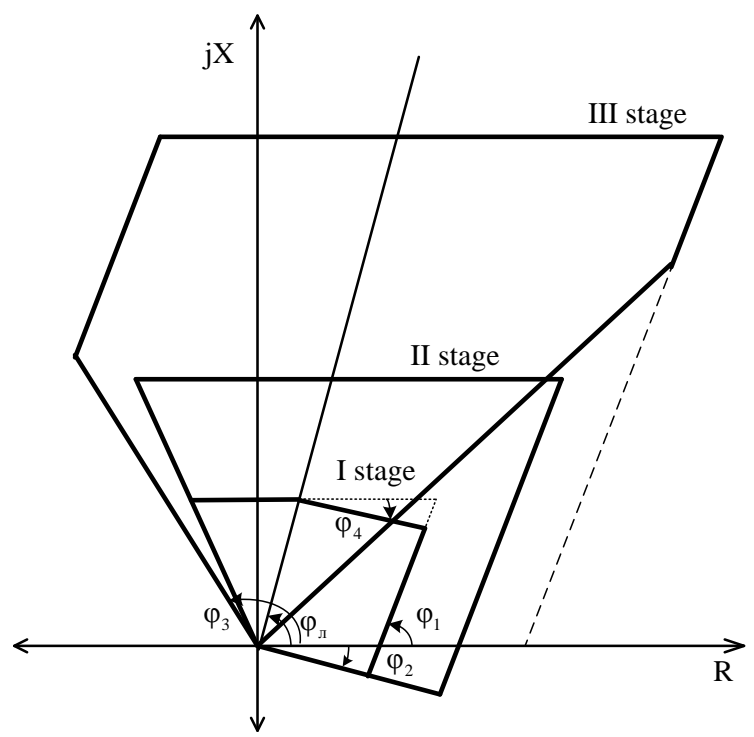

\section{Figure 1. Characteristic of High-voltage Line Digital Distance Relay Protection}

With external circuit on the previous line, the decrease of resistance measurement leads to the hit of this resistance vector in field characteristic, and as result, the excessive trigger will appear at the end protected line. In addition, there is an increase of the active resistance due to its recharge from other sources that is connected to opposite ends of the line. This resistance is part of the resistance measurement, and therefore increases the last, but not towards the top and side face of characteristic, which possibly makes the measured vector output of scope characteristic. To ensure the capture of the transient resistances, characteristic should be extended to higher resistances, notably right lateral face should be referred to the right. This extension of the characteristic increases the possibility of excessive action at the sending end of the line in through an operating current. The two analyzed factors improve the resistance then for increasing efficiency distance relay protection requires to reduce the scope of the characteristic. To carry out the reduction by shifting the right-hand side edge of the parallelogram in the direction of reducing the resistance is impossible, since leads to a reduction in scope and, therefore, reduce the capture of transient resistance in this area. Therefore, reducing the scope of the characteristic operates through bevel the top edge of the parallelogram in the direction of the lower bound, from the point of intersection of the upper bound of the ray from the origin at an angle of resistance to the protected line. Bevel the top edge is in the form of a straight line with a negative angle relative to the $\mathrm{X}$-axis (upper bound).

The increase of resistance measurement results is caused by operating current when the faults at the start line. This causes the output of the measured resistance vectors beyond the left characteristic face, which causes the necessarily to expand left side characteristic in the second quadrant, to ensure the efficiency of the distance relay protection while faults are in the beginning of the line (including the part of line between the transformers current at each end of the line and buses). However, at low operating currents, the measure would lead to excessive actions when faults on adjacent lines (behind relay). To reduce this effect, the angle $\varphi_{3}$ of the phase-limiting line should put greater $90^{\circ}$, but within no more than $120-130^{\circ}$.

The analysis shows that expanding the scope of the characteristic in the second quadrant and the reduction of characteristic due to its slant in the first quadrant, along with a reduction in false actions in the first case and excessive actions in the second case could result in low operating currents through the losses of the opposite character from 
configuration characteristic: excessive actions in the first case and the false actions in the second case. Counting for these losses and optimization of the losses of distance relay protection is quite possible with the developed probabilistic methods [1,2]. In this article, this problem is not posed. Since these functional losses depend on the value of operating current, low loss will be achieved under different settings characteristic resistance relay. Therefore it is expedient and necessary in the measuring resistance relay provide automatic control of the channel configuration characteristic. If this is not possible to estimating the reduction in technical efficiency of distance relay protection, notably losses, during the deformation of characteristic to detune from the previous operating mode and to increase sensitivity. It seems logical enough to take the area ratio criterion characteristics due to its deformation to the base area of a parallelogram form-building in the complex impedance surface.

Functioning and setting distance relay protection is currently using in expertmanagement method does not allow to quantify the quality of the operation of this protection in the electrical network. Therefore, the criterion technical efficiency is needed to uniquely identify the setting and estimating the quality of distance protection. For backup stages distance relay protection, resistance settings are determined by the detuning of the load mode, or on the basis of sensitivity and time setting is usually determined by the opposite-step basis. So this criterion is especially important for the backup stages distance relay protection, a final setting which satisfies the selectivity and sensitivity in complex networks in some cases is not possible (for example, in the presence of bypass shunt links the protected object), and requires an assessment of options is not enough full settings at the time of action to choose the best option. The criterion in this case can serve as indicators of technical efficiency and its components. Option with the maximum of technical effect and technical efficiency, or minimum total losses may be taken as the optimum option.

Losses of false actions in the working conditions (operating conditions) are less dependent on the characteristics of the network, notably regime-switching network conditions and types of faults. The last due to the fact, that the distance protection equipment is performed separately from the inter-phase and single-phase faults. There are depending on the setting stage values, which form the boundaries of the space on the protected line. The analysis shows that, the losses of false actions are disproportionately large compared with the refusals of work and excessive actions and are practically independent of the functional characteristics of the network (regime-switching network status and type of faults). It is therefore advisable to assess the best option for refusals of work and excessive actions against false operations. Thus the greatest difficulty is the definition of excessive actions backup stages, which may depend on the actions and failures of backup stages lines in the direction of interested backup stages.

As opposed to the basic stages, backup stages of distance relay protection of high voltage lines, as indicated above, in the functioning part set up independently of each other (by the offset of the operating modes, or on the basis of sensitivity) and time setting for each interested stage of we recommend choose by the opposite-step basis, notably by increasing the value of time selectivity of the considered backup stages compared with the time setting of backup stages on previous lines. The called algorithm for setting backup stages in the complicated structure networks can lead to inconsistency in the parameter sensitivity of the response and the duration of the interested stage lines of the network steps in the direction of interested backup stages.

In order to definitely and correctly consider the excessive actions considered backup stage of the line distance relay protection should be identified cooperative section in the operative direction with the backup stage protection of the peripheral lines. The algorithm for identifying excessive section is separated into different cases: the superiority of the sensitivity of the considered backup stage with controlled backup stages of the previous lines; and the superiority of the controlled backup stages with the considered backup 
stage. In these cases, the time setting of the considered backup stage may be more, less, equal to the time setting of the controlled backup stages and should be regarded as a full aspect of the setting.

The implementation of these algorithms requires providing a number of logic due to technical efficiency and loss of the considered backup stage, view, monitor and identify interactive sections between the considered backup stage and the controlled backup stage. This logic is determined by the parameter response and time settings of the considered backup stage protected line and the controlled backup stage network in the direction of the considered backup stage. Therefore, the algorithm must always control the parameter response and time settings of these backup stages. In this case the protection of transformers and autotransformers network is excluded, because these backup stages directed to the adjacent network, practically the same in total-summarized the action of backup stages adjacent lines in the circuit networks in the space of these lines. This allows identifying specific sections of cooperation under the consideration and controlled backup stages of these lines and, therefore, to estimate the excessive losses of specific interactions between the considered and controlled backup stages of all adjacent line networks, adjacent to all sides of the network transformer or autotransformer. Discussed the interaction can be expressed by the following theses.

1. If possible joint actions between the considered and controlled backup stages on the whole space peripheral lines, notably the boundaries of the considered and controlled backup stages are far from the ends of these lines in the direction of the considered backup stage, then when the time setting of the considered backup stage is less than the controlled backup stage, all the joint actions will be excessive actions, (because measuring relay of the controlled backup stages will not have time to operate). When the time setting of the considered backup stage is equal to the controlled backup stage then this will take place synchronous actions between the considered and controlled backup stages. A half of this should be considered as excessive actions of the considered backup stage. When the time setting of the considered backup stage is more than the controlled backup stage then excessive actions of the considered backup stage will not be.

2. If the boundary of the considered backup stage is placed on the space of peripheral lines, and the controlled backup stages are more sensitive, then operation of the considered backup stage will be the same as in point 1 .

3. If the boundary of the considered backup stage on the space of peripheral lines, and the considered backup stage is more sensitive than the controlled backup stage then the section from boundary (the controlled backup stage) to boundary (the considered backup stage) will be failure actions of the controlled backup stage (equivalent the excessive actions of the considered backup stage). And the section from the beginning of peripheral lines to the boundary of the considered backup stage will be possible joint actions between the considered and controlled backup stages as in point 1 .

The visual representation of the above interacting backup stages (the parameter response and time setting) contributes to the structure of the scheme wiring area highvoltage electrical network shown in Figure 2. In the scheme, the numeral marked nodes in the network, and its components: the protected line №, elements in the direction of the considered backup stage №, 1 : the first with periphery relative to this stage $1 \Pi$, the second $2 \mathrm{p} 1,2 \mathrm{p} 2,2 \mathrm{p} 3,2 \mathrm{p} 4$, the third $3 \mathrm{p} 1,3 \mathrm{p} 2,3 \mathrm{p} 3$ and the fourth $4 \mathrm{p} 1,4 \mathrm{p} 2$ peripheries. At the ends of lines, transformers and autotransformers are switches designated by the numbers 1 and 2 for the two-winding transformers and lines, 1, 2 and 3 for auto-transformers. Stepto-step distance relays protection, including the backup stages at the ends of lines and equipment are identified findings docked protected designations of force elements and switches, for example, as mentioned above are backup stage №, 1. Shown in the scheme is a radial network with the exception of the bypass communication, shunting the protected 
line № and the first $1 p=4$, the second $2 p 4=1$ second, and third peripheral lines $3 p 3=1$. Determination time setting of backup stages in the radial part of the network depending on the development in each direction of its structure, such as the considered backup stage №, 1, it gives a different value of time. The highest value of time is taken as setting, which is unlikely to be effective. And another unique selection criterion for defining time setting of the backup stage is not available If this attempt to supplement the selection of time setting backup stage protection №, 1 by the opposite-step principle for by-pass lines, can see that the time setting of backup stage protection №,1 will grow from the previously value in the radial network. There is no logical due to the uniqueness criterion in the opposite-step principle, when it is not, and the question remains open.

Analysis and calculation of technical efficiency for different time settings of each stage allows selecting an acceptable option. The analysis and calculations can be performed on the developed probabilistic methods and algorithms for estimating the technical efficiency of distance relay protections in high-voltage networks [3, 4, 7, 8, and 9]. Technical effect for back-up zone (stage) of distance relay protection (Fig.2) can calculate by (1):

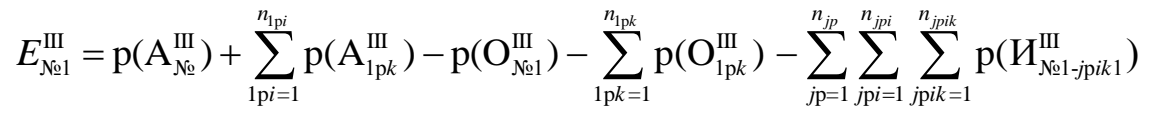




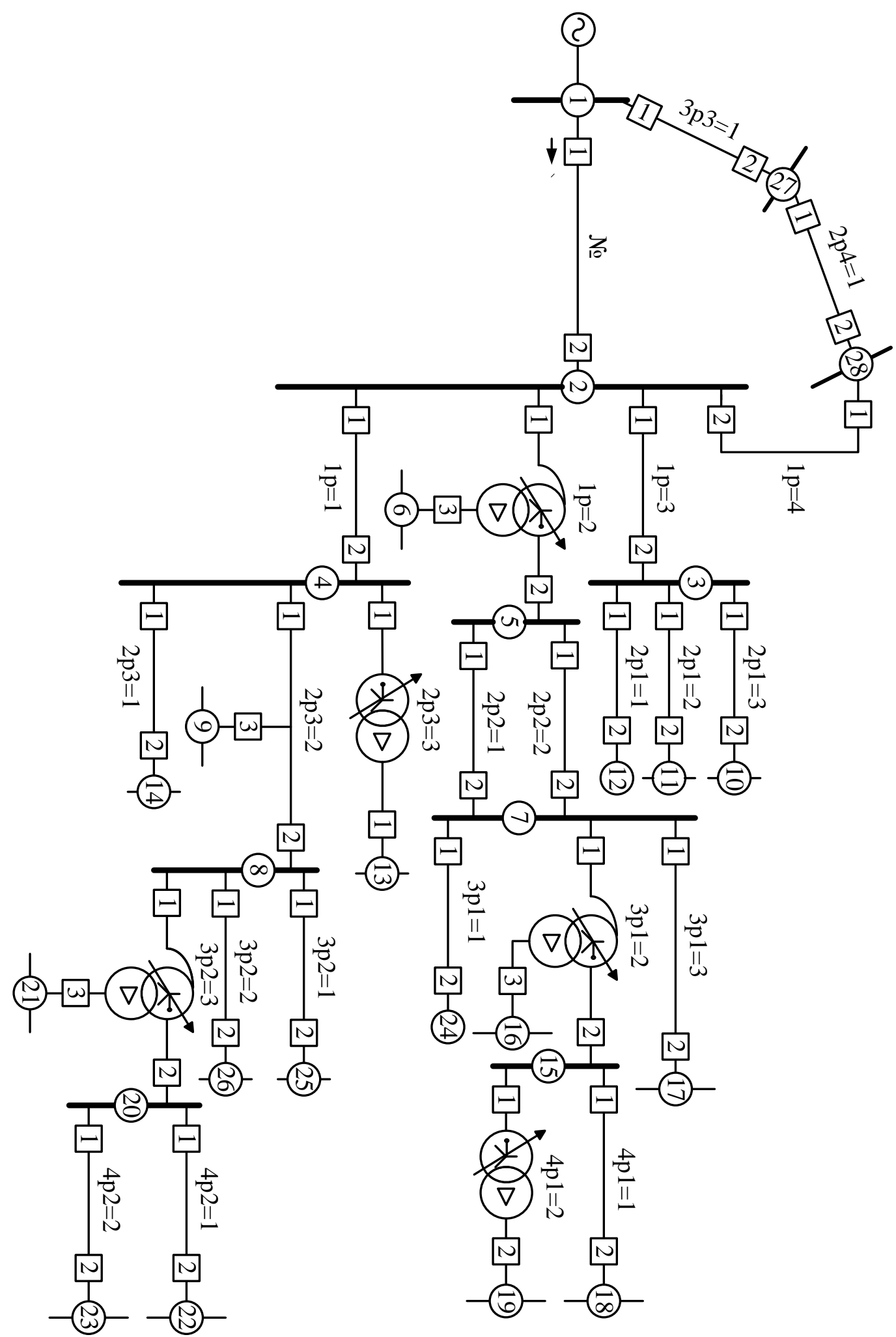

Figure 2. The Structure of a Given Network (as an example for choosing backup stage setting of the considered distance relay protection at the end 1 of the line №)

Where the lower indexes: № - protected line, $\mathrm{p}$ - previous lines (elements), pp previous (elements) of previous lines (elements) (Fig. 2). The letter p designates 
probabilities of events: A - faults on a protected line, $\mathrm{O}$ - operation refusals, И excessive actions; I - first stage, II - second stage, III - back-up stages of line distance relay protection.

Probability faults $\mathrm{p}(\mathrm{A})$ for back-up (third) stage:

$$
\mathrm{p}\left(\mathrm{A}_{N_{\mathrm{No}}}^{\mathrm{III}}\right)+\sum_{1 \mathrm{p} i=1}^{n_{\mathrm{lp}}} \mathrm{p}\left(\mathrm{A}_{1 \mathrm{p} k}^{\mathrm{III}}\right)=\omega_{\text {№ }} \mathrm{m}\left(T_{N_{\mathrm{No}}}^{\mathrm{III}}\right)+\sum_{1 \mathrm{p} i=1}^{n_{\mathrm{lp}}} \omega_{1 \mathrm{pk}} \mathrm{m}\left(T_{1 \mathrm{p} k}^{\mathrm{III}}\right)
$$

Where $\omega_{1 p k}-$ the flow parameter of fault types on 1pk-th line,

$\mathrm{m}\left(T_{\mathrm{No}}^{\mathrm{III}}\right), \mathrm{m}\left(T_{\mathrm{lpk}}^{\mathrm{III}}\right)$ - average duration of detection (lock) fault channels of the third stages (third stage time setting)

Mechanism of excessive actions of the third stages is similar formation of excessive action of the second stage. However, unlike the main stages in which the same names delay stages are almost identical, in meshed networks setting time of the back-up stages may be different: setting time of the back-up stage of considered protected line and backup stages of the peripheral elements may be equal or greater than the considered back-up stage of the protected line stage. Therefore it is necessary to consider separately these cases mentioned by the time the interaction with all elements of the network:

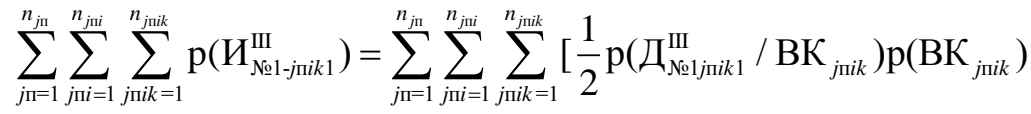

$$
\begin{aligned}
& \left.+\mathrm{p}\left(\mathrm{O}_{\text {Nol } 1 \text { IIIik } 1}^{\mathrm{III}} / \mathrm{BK}_{\text {jпik }}\right) \mathrm{p}\left(\mathrm{BK}_{\text {jпik }}\right)\right]
\end{aligned}
$$

Based on the above, the above analysis for the backup stages of distance relay protection lines may be implemented as a dynamic intelligent software that provides view of network topology, setting by the parameter response (current, resistance) and by the time of the considered and controlled backup stages in the direction of each considered backup stage line. Backup stage transformers and autotransformers at the same time can be omitted. Refusals of work and action of the controlled backup stage lead to excessive actions of the considered backup stage line, defined by setting boundaries (zones of action) by parameter response of the considered and every controlled stages on space lines of the network that correspond to the direction of the considered backup stage. As a result of considering are identified and calculated the probability of refusals of work and action controlled backup stage on network peripheral lines, and according to the probabilities excessive actions of the considered backup stage are probably defined.

\section{Conclusions}

1. Clear and objective criteria for determining time setting of the backup stage of distance relay protection of the hard-closed electrical network is excessive actions of technical efficiency, which allows choosing the optical option.

2. For practical usage of the named criterion of excessive action is appropriate development program for the formation and optimization options for setting each backup stage with modules calculations in the parameter response and time setting, identify and minimize excessive actions of every backup stage by refusal of work and action of the controlled backup stage in the direction of the considered backup stage.

3. In criteria of the technical effect and the technical efficiency of distance relay protection is necessary to include losses that accompany deformation characteristic resistance relay (which are used to achieve different interested effects). 


\section{References}

[1] Fedoseev A. M. Relay protections power system. M.:Energoatomizdat, 1984. P.520.

[2] A.V. Shmoilov "Probability technologies in electric power industry," Proc. 6-th Russian-Korean Intern. Symp. On Science and Technology KORUS-2002, vol.2, pp.421-424, 2002.

[3] A.V.Shmoilov, L.V.Krivova, E.I.Stoyanov, K.V. Ignatiev, "Probabilistic method select of the borders interval data for electroenergetic problems," Proc.THE HIGH SCHOOL "Problems of energy, No 7-8/1, pp.146-157, 2008.

[4] A.F Prutik., A.V. Shmoilov, "Setting-up algorithms of relay protection," The Forth International Forum on Strategic Technology (IFOST 2009), pp. 45-50, 2009.

[5] Schneerson E.M. Digital relay protection. - M. Energoatomizdat, 2007, P.549.

[6] Nudelman G.S., Shalin A.I. Microprocessor-based relay protection. News of electrical engineering. 2008. - P. 74-79.

[7] Prutik A.F., Tran Minh, Shmoilov A.V. The selectivity and the technical efficiency of relay protection and automatics. Journal "Energy problems", 2010, P.154-163.

[8] Shmoilov A.V. Probability technologies in electric power industry. Proc. 6-th Russian-Korean Intern. Symp. On Science and Technology KORUS-2002, Novosibirsk. - 2002. - Vol.2 - P.421-424.

[9] Tran Hoang Quang Minh, Nguyen Trung Thang. Criterion Technical Efficiency of Line Distance Relay Protection, International Journal of Advanced Science and Technology, Vol. 53, 2013, P. 123-129.

\section{Author}

Tran Hoang Quang Minh defended his PhD thesis at Tomsk Polytechnic University, Tomsk City, Russian Federation. The author's major fields of study are High-voltage Power System and Relay Protections. He is working as Lecturer in Faculty of Electrical and Electronic Engineering, Ton Duc Thang University, Ho Chi Minh City, Vietnam. 
International Journal of Signal Processing, Image Processing and Pattern Recognition Vol.8, No.9 (2015) 